\title{
Armodafinil improves wakefulness and long-term episodic memory in nCPAP-adherent patients with excessive sleepiness associated with obstructive sleep apnea
}

\author{
Thomas Roth • Gregory A. Rippon • Sanjay Arora
}

Published online: 15 September 2007

(C) Springer-Verlag 2007

\begin{abstract}
Residual excessive sleepiness (ES) and impaired cognition can occur despite effective and regular nasal continuous positive airway pressure (nCPAP) therapy in some patients with obstructive sleep apnea (OSA). A pooled analysis of two 12-week, randomized, double-blind studies in nCPAP-adherent patients with ES associated with OSA evaluated the effect of armodafinil on wakefulness and cognition. Three hundred and ninety-one patients received armodafinil (150 or $250 \mathrm{mg}$ ) and 260 patients received placebo once daily for 12 weeks. Efficacy assessments included the Maintenance of Wakefulness Test (MWT), Cognitive Drug Research cognitive performance battery, Epworth Sleepiness Scale, and Brief Fatigue Inventory. Adverse events were monitored. Armodafinil increased mean MWT sleep latency from baseline to final visit by $2.0 \mathrm{~min}$ vs a decrease of $1.5 \mathrm{~min}$ with placebo $(P<$ $0.0001)$. Compared with placebo, armodafinil significantly improved quality of episodic secondary memory $(P<0.05)$ and patients' ability to engage in activities of daily living $(P<0.0001)$ and reduced fatigue $(P<0.01)$. The most common adverse events were headache, nausea, and insomnia. Armodafinil did not adversely affect desired nighttime sleep, and nCPAP use remained high (approximately $7 \mathrm{~h} /$ night). Adjunct treatment with armodafinil significantly improved wakefulness, long-term memory,
\end{abstract}

\section{T. Roth $(\bowtie)$}

Henry Ford Hospital,

2799 West Grand Blvd, CFP-3,

Detroit, MI 48202, USA

e-mail: Troth1@hfhs.org

G. A. Rippon · S. Arora

Cephalon, Inc.,

41 Moores Road,

Frazer, PA 19355, USA and patients' ability to engage in activities of daily living in nCPAP-adherent individuals with ES associated with OSA. Armodafinil also reduced patient-reported fatigue and was well tolerated.

Keywords Armodafinil · Fatigue - Obstructive sleep apnea syndrome $\cdot$ Sleepiness $\cdot$ Wakefulness

\section{Introduction}

Obstructive sleep apnea (OSA) syndrome includes excessive sleepiness (ES), fatigue, sleep fragmentation, impaired alertness, cognitive dysfunction, and mood disturbances [1, 2]. OSA is typically characterized by recurrent complete or partial airway collapse during sleep, resulting in frequent apnea and hypopneic events lasting at least $10 \mathrm{~s}$ [1]. Although the reported prevalence of OSA in the general population varies as a function of diagnostic criteria [2], OSA syndrome has been estimated to occur in 4 and $2 \%$ of middle-aged North American men and women, respectively [3]. OSA can lead to serious medical, social, and public safety consequences, and patients are at an increased risk for cardiovascular disease [4] and motor vehicle and occupational accidents [5, 6]. Quality of life and social functioning are also significantly impaired in this patient population [7, 8]. Fatigue, in particular, can contribute to reduced quality of life [1,9]; thus, the assessment of patient-rated fatigue is an important outcome to evaluate and monitor in patients with OSA.

Nasal continuous positive airway pressure (nCPAP) therapy is the recommended standard of care for treating patients with moderate to severe OSA [10]. In a comprehensive review of 36 clinical studies, nCPAP therapy was shown to effectively reduce objective and subjective 
measures of sleepiness and improve quality of life in patients with moderate and severe OSA [11]. However, despite receiving adequate $\mathrm{nCPAP}$ therapy, half of patients with OSA still experience residual ES as measured by objective assessments as well as impairments in mood or cognition [12-18]. Cognitive impairment in patients with OSA is characterized by deficits in attention, learning and memory, and executive function (planning and problem solving) and may persist despite nCPAP therapy [12, 1517]. A review of the literature suggests that while nCPAP therapy can substantially improve quality of life for patients with OSA, it has a smaller effect on improving cognitive performance [7].

Modafinil, a racemic mixture consisting of equal amounts of $R$ - and $S$-enantiomers, has been shown to improve wakefulness in patients with OSA who experience residual ES despite regular nCPAP use [19, 20]. Pharmacokinetic studies have shown that the half-life of $R$-modafinil (10$14 \mathrm{~h}$ ) is significantly longer than the half-life of $S$-modafinil (3-4 h) [21-23]. Armodafinil has higher plasma concentrations later in the day compared to modafinil on a "milligram-to-milligram" basis and improves healthy subjects' wakefulness and ability to sustain attention for a longer period of time compared with modafinil [24]. Armodafinil has also recently been shown to improve wakefulness in patients with ES associated with narcolepsy in a 12-week double-blind, placebo-controlled study [25].

Recent findings from two 12-week, double-blind, placebocontrolled studies of similar design indicate that armodafinil improved wakefulness and overall clinical condition in nCPAP-adherent patients with OSA and residual ES [26, 27]. In the present pooled analysis of the two OSA studies $[26,27]$, the effects on wakefulness, cognition, and fatigue and the safety and tolerability of armodafinil compared with placebo were evaluated in OSA patients adherent to nCPAP therapy.

\section{Materials and methods}

\section{Study design and procedures}

Data from two 12-week, multicenter, double-blind, placebocontrolled, parallel-group clinical studies were pooled for analysis. The pooling of these data was based on similarities in patient population, study design, and treatment duration in the two studies [26, 27]. One study was conducted in 36 centers across the USA, Australia, Russia, Germany, and France [26], and the other study was conducted in 37 centers across the USA and Canada [27]. The study protocols were approved by an Independent Ethics Committee or Institutional Review Board at each center. Both studies were conducted in accordance with the Good
Clinical Practice: Consolidated Guideline [28] and national and local laws and regulations.

Patients were randomized to receive armodafinil $150 \mathrm{mg}$ $(n=129)$ or placebo $(n=130)$ daily in the first study [26] and armodafinil $150(n=131)$ or $250 \mathrm{mg} /$ day $(n=131)$, or placebo $(n=130)$ in the second study [27]. Study drugs were initiated with a dose of $50 \mathrm{mg} /$ day on day 1 and then increased in increments of $50 \mathrm{mg}$ starting on day 2 and every 2 days thereafter until the assigned dose was reached (i.e., $150 \mathrm{mg}$ /day on day 4 for both studies or $250 \mathrm{mg} /$ day on day 8 for the second study). The study drugs were taken once daily in the morning (before 08:00 and approximately $30 \mathrm{~min}$ before breakfast). Monitoring of patients' compliance with study drug administration was the responsibility of the investigator at each center and was assessed by completed study drug accountability records and reviews of patient diaries. nCPAP use at home was also monitored objectively by investigators at each visit to the study center, using the REMstar ${ }^{\mathbb{B}}$ Auto CPAP System (Respironics, Murrysville, PA). The REMstar Auto CPAP System was used in CPAP mode.

\section{Patient selection criteria}

Methods for selecting patients in both studies have been described previously [26, 27]. Men and women 18 to 65 years of age with a current diagnosis of OSA as defined by the International Classification of Sleep Disorders [1] were included in both studies. Patients were at least moderately ill (Clinical Global Impression of Severity of Illness rating greater than or equal to 4) [29] and had a complaint of ES. Patients were eligible if ES was determined to be pathological, based on an Epworth Sleepiness Scale (ESS) score greater than or equal to 10 [30]. All patients were receiving stable (greater than or equal to 4 weeks) and effective nCPAP therapy on a regular basis (greater than or equal to $4 \mathrm{~h}$ per night on greater than or equal to $70 \%$ of nights) during the 2 -week evaluation period. The CPAP use was monitored using the REMstar Auto CPAP System. Effectiveness of therapy was determined by an apnea-hypopnea index (AHI) less than or equal to ten events per hour on nighttime polysomnography (PSG).

\section{Patient exclusion criteria}

Patients were excluded if they had any of the following: a diagnosis of a sleep disorder other than OSA, symptoms of ES associated with a clinically significant uncontrolled medical or psychiatric condition as determined by the investigator, any disorder that might interfere with drug absorption, distribution, metabolism, or excretion, a history of drug or alcohol abuse, as determined by the Diagnostic 
and Statistical Manual of Mental Disorders IV criteria [31] or by a positive result from the urine drug screen given at screening and again at the final visit, caffeine consumption greater than $600 \mathrm{mg} / \mathrm{day}$, or a clinically significant sensitivity to central nervous system stimulants or modafinil. Women who were pregnant or breast feeding were also excluded. The use of any substance that could affect wakefulness or sleepiness (e.g., modafinil, sodium oxybate, melatonin, lithium, St. John's wort, methylphenidate, amphetamines, pemoline, antipsychotic agents, benzodiazepines, zolpidem, anticonvulsants, or barbiturates), use of other excluded agents (e.g., monoamine oxidase inhibitors, anticoagulants), use of clinically significant amounts of nonprescription drugs within 7 days of screening, and use of investigational drugs within 1 month of screening was prohibited. All patients provided written informed consent before participation in these studies and were compensated for their participation.

\section{Assessments}

\section{Efficacy assessments}

Efficacy assessments were performed for both studies at baseline and weeks 4,8 , and 12 . The primary efficacy variables in the two individual studies were the change from baseline to final visit (week 12 or last postbaseline measurement) in mean Maintenance of Wakefulness Test (MWT) [32, 33] sleep latency averaged across the first four tests and the proportion of patients with at least minimal improvement on the Clinical Global Impression of Change (CGI-C) [29]. The MWT was conducted as six separate 30-min sessions at 09:00, 11:00, 13:00, 15:00, 17:00, and 19:00. Sleep latency was defined as the time to onset of the first of three consecutive epochs of stage 1 sleep or the time to onset of any epoch of stages 2, 3, and 4 sleep or rapid eye movement sleep [34]. Sleep latencies were averaged across the first four tests $(09: 00,11: 00,13: 00,15: 00)$ and the last three tests $(15: 00,17: 00,19: 00)$ to distinguish between early- and late-day effects, respectively. The proportion of patients with at least a minimal improvement on the CGI-C was assessed to determine patients' overall clinical improvement in the individual studies; however, these data were not poolable for this analysis because of a substantial difference in the percentage of responders to placebo between the two studies.

The Cognitive Drug Research (CDR) battery of tests [35, 36] was administered by computer as six separate 25 -min sessions conducted at 09:30, 11:30, 13:30, 15:30, 17:30, and 19:30. The CDR battery includes five memory tests (immediate word recall, delayed word recall, numeric working memory, word recognition, and picture recognition) and three attention tests (simple reaction time, choice reaction time, and digit vigilance). Two composite factors are derived from the memory tests in the CDR: quality of episodic secondary memory, a measure of long-term memory that measures the ability to recall verbal and visual information, and speed of memory, which assesses the time it takes to decide whether information is held in memory. Two composite factors are derived from the CDR for attention: power of attention, which measures the ability to focus attention and avoid distraction (concentration), and continuity of attention, which measures the ability to sustain attention (vigilance). Similar to the MWT, the CDR scores were averaged across the first four test sessions $(09: 30,11: 30,13: 30,15: 30)$ and the last three test sessions $(15: 30,17: 30,19: 30)$ to assess cognitive effects on earlyand late-day measurements.

The patients' ability to engage in daily activities was assessed at each visit by the ESS [30]. ESS total scores range from 0 to 24 , with higher scores indicating greater sleepiness. An ESS score greater than or equal to 10 indicates pathological sleepiness [30]. The severity and impact of patient-rated fatigue on daily functioning were assessed at each visit by the nine-item Brief Fatigue Inventory (BFI) [37] and were based on changes in global fatigue (average of all nine questions) and worst fatigue in the past $24 \mathrm{~h}$ (item 3). The rating scale for the BFI ranges from 0 (no fatigue) to 10 (as bad as you can imagine). A score of greater than or equal to 7 for either global fatigue or worst fatigue in the past $24 \mathrm{~h}$ is indicative of severe fatigue. Both the ESS and BFI were administered before the first MWT session (09:00) at each visit.

\section{Safety and tolerability assessments}

Adverse events were monitored and recorded by the study investigators at each center throughout both studies. Clinical laboratory tests (blood chemistry, hematology, urinalysis), vital signs (resting heart rate and systolic and diastolic blood pressure 3 and $13 \mathrm{~h}$ postadministration), and electrocardiograms were obtained at screening, baseline, and weeks 4, 8, and 12. Physical examinations were performed at baseline and week 12. Patients' use of nCPAP was monitored at least 2 weeks before baseline and throughout both studies using the Respironics REMstar Auto CPAP system. Effect on nighttime sleep was determined by PSG, which was performed the night immediately after the measurement of daytime MWT during the second screening visit and the final visit. The PSG was conducted for $8 \mathrm{~h}$, starting within $30 \mathrm{~min}$ of the patient's usual bedtime but not earlier than 21:30.

Clinically significant elevations in resting systolic and diastolic blood pressure were defined a priori as greater than or equal to $140 \mathrm{mmHg}$ with an increase of greater than or equal to $10 \%$ and greater than or equal to $90 \mathrm{mmHg}$ with 
an increase of greater than or equal to $10 \%$, respectively. Patients with worsening hypertension included those who had a history of hypertension at baseline and who started new antihypertensive medication and/or increased the dose of previously used antihypertensive medication during the studies. Patients with newly diagnosed hypertension included those who had no history of hypertension at baseline and who started antihypertensive medication during the studies. Patients at risk for hypertension included those who had at least two clinically significant elevations in blood pressure readings between baseline and final visit.

Statistical analysis

Descriptive statistics were used to summarize continuous and categorical demographic variables. Efficacy assessments were analyzed at weeks 4,8 , and 12 using observed cases and at final visit (week 12 or last postbaseline measurement) using the last observation carried forward approach. Efficacy analyses included randomized patients who received at least one dose of study drug and had a baseline measurement with at least one postbaseline measurement on the MWT and CGI-C. Safety and tolerability analyses included all randomized patients who received at least one dose of study drug. All efficacy assessments were analyzed by analysis of variance (ANOVA) with treatment and study as factors. Tests of poolability for all continuous efficacy variables across the two studies were conducted using an ANOVA with treatment and study and treatment by study interaction as factors. Vital signs and data from nighttime PSG were analyzed by Wilcoxon rank-sum test. For nCPAP use, the change from baseline to on-treatment values was analyzed using one-way ANOVA. All statistical tests were twotailed, and the $5 \%$ level of significance was used.

\section{Results}

Patient demographics and disease characteristics

Of 658 patients randomized, 651 were included in the safety analysis, and 601 were evaluable for efficacy (Fig. 1). Patient demographics and disease characteristics for all patients in the safety analyses are summarized in Table 1. The mean body mass index was $36.6 \mathrm{~kg} / \mathrm{m}^{2}$. Patients reported high nightly nCPAP usage (mean [SD] hours of nightly use, armodafinil 6.9 [1.2] h; placebo 6.9 [1.0] h). The nCPAP therapy was effective, as shown by mean (SD) AHI values of 1.5 (3.3) and 1.2 (2.1) events/h in the armodafinil and placebo groups, respectively. Overall, $41 \%$ of patients had a history of hypertension at baseline.

\section{Effects on wakefulness}

Mean (SD) sleep latency at baseline across the first four MWTs (09:00, 11:00, 13:00, 15:00) was 22.8 (8.4) and 23.2 (7.9) $\mathrm{min}$ for the armodafinil and placebo groups, respectively. At all study visits, armodafinil significantly improved mean sleep latency across the first four MWTs

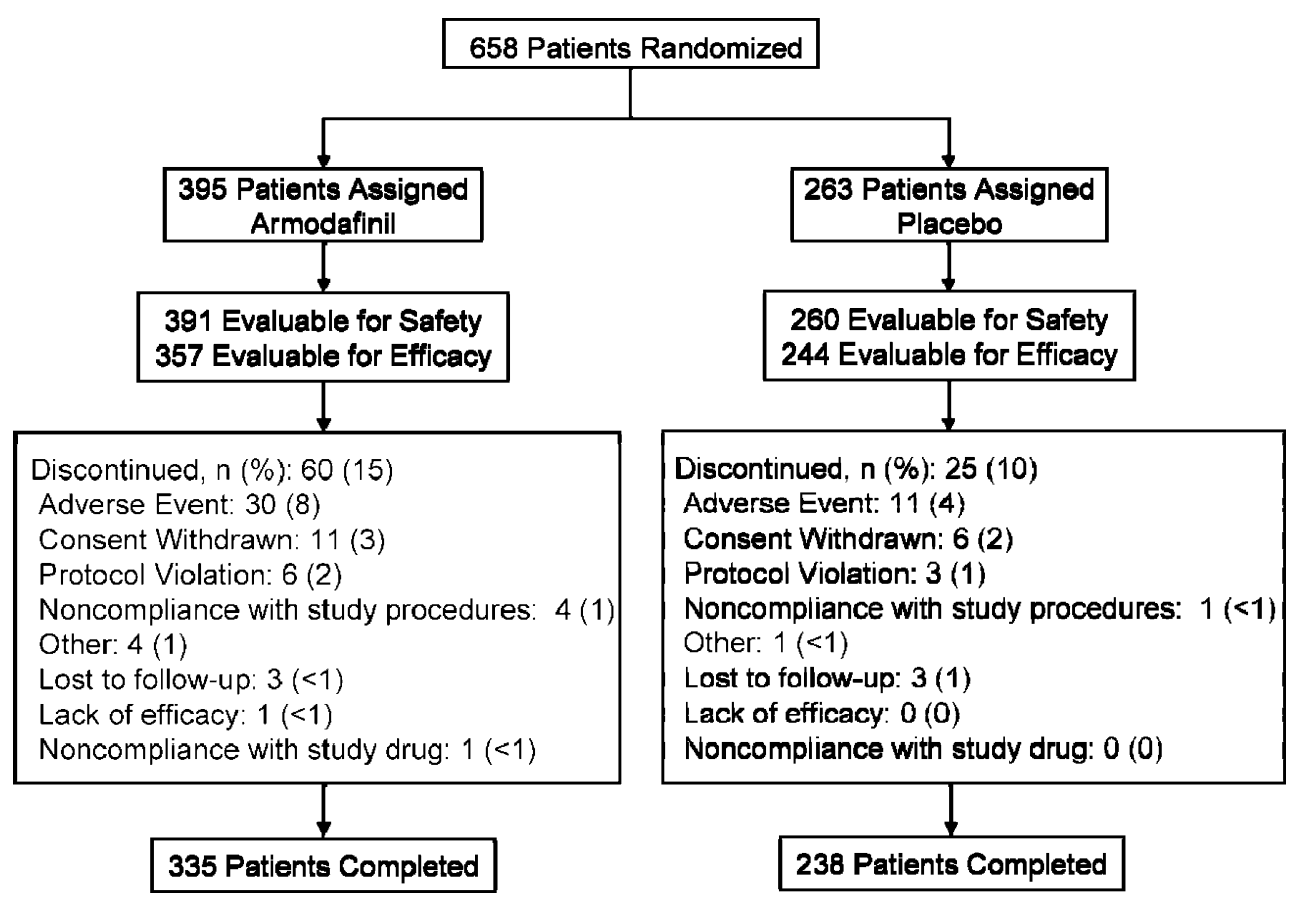

Fig. 1 Patient disposition 
Table 1 Patient demographics and baseline characteristics

\begin{tabular}{lll}
\hline Characteristic & $\begin{array}{l}\text { Armodafinil } \\
(n=391)\end{array}$ & $\begin{array}{l}\text { Placebo } \\
(n=260)\end{array}$ \\
\hline $\begin{array}{ll}\text { Age (years) } \\
\text { Mean (SD) }\end{array}$ & $49.7(9.0)$ & $50.3(9.1)$ \\
Sex, $n$ (\%) & $283(72)$ & $183(70)$ \\
Men & $108(28)$ & $77(30)$ \\
Women & & \\
Race, $n(\%)$ & $327(84)$ & $224(86)$ \\
White & $36(9)$ & $21(8)$ \\
Black & $6(2)$ & $3(1)$ \\
Asian & $22(6)$ & $11(4)$ \\
Other & 0 & $1(<1)$ \\
Missing & & $111.2(23.7)$ \\
Weight (kg) & $110.4(24.6)$ & \\
Mean (SD) & & $36.9(7.5)$ \\
BMI (kg/m $\left.{ }^{2}\right)$ & $36.4(8.0)$ \\
Mean (SD) & & $138(53)$ \\
CGI-S, $n(\%)$ & $219(56)$ \\
Moderately ill & $172(44)$ & $122(47)$ \\
Markedly, severely, or extremely ill & & \\
nCPAP (h) & & \\
Mean (SD) & $6.9(1.2)$ & $6.9(1.0)$ \\
AHI & & \\
Mean (SD) & $1.5(3.3)$ & $1.2(2.1)$ \\
History of hypertension & & \\
$n$ (\%) & & \\
\hline
\end{tabular}

AHI Apnea-hypopnea index, BMI body mass index, CGI-S Clinical Global Impression of Severity of Illness, $n C P A P$ nasal continuous positive airway pressure

compared with placebo (Fig. 2a). The mean change from baseline in sleep latency at final visit was 2.0 min for the armodafinil group compared with -1.5 min for the placebo group $(P<0.0001)$.

Armodafinil also improved patients' ability to maintain late-day wakefulness (MWTs at 15:00, 17:00, 19:00) compared with placebo (Fig. 2b). Mean (SD) sleep latency at baseline across the last three MWTs was 24.4 (7.8) and 24.6 (7.4) $\mathrm{min}$ for the armodafinil and placebo groups, respectively. The mean change from baseline in sleep latency at final visit was $1.1 \mathrm{~min}$ for the armodafinil group compared with $-0.3 \mathrm{~min}$ for the placebo group $(P<0.05)$.

Effects on memory and attention

Compared with placebo, armodafinil significantly improved the quality of episodic secondary memory across the first four tests $(09: 30,11: 30,13: 30,15: 30)$ at all study visits $(P<0.05$; Fig. 3a). The mean (SD) change from baseline in quality of episodic secondary memory at final visit was 10.2 (31.7) U for the armodafinil group compared with -0.7 (46.3) $\mathrm{U}$ for the placebo group $(P<0.01)$. Differences between the treatment groups did not achieve statistical significance at the final visit for speed of memory, power of attention, or continuity of attention across the first four tests.

Across the later three tests of the day $(15: 30,17: 30$, $19: 30$ ), the difference in the quality of episodic secondary memory was statistically significant at week 8 for armodafinil compared with placebo $(P=0.011$; Fig. $3 b)$, with a trend toward significance at week $12(P=0.081)$. The difference between treatment groups was not statistically significant at the final visit for the quality of episodic secondary memory. There were no significant changes from baseline to final visit between the armodafinil and placebo groups for speed of memory, power of attention, or continuity of attention across the last three tests.

Effects on patients' ability to engage in activities

The mean (SD) ESS scores were high at baseline (15.4 [3.5] and 15.9 [3.5] for the armodafinil and placebo groups, respectively), despite the high nCPAP use as shown in Table 1. Compared with placebo, treatment with armodafinil significantly improved patients' ability to engage in activities of daily living (ESS) at all visits $(P<0.0001$; Fig. 4). Nearly half of all patients $(49 \%)$ in the armodafinil group responded to treatment (total ESS score less than 10 at final visit) compared with $26 \%$ in the placebo group.
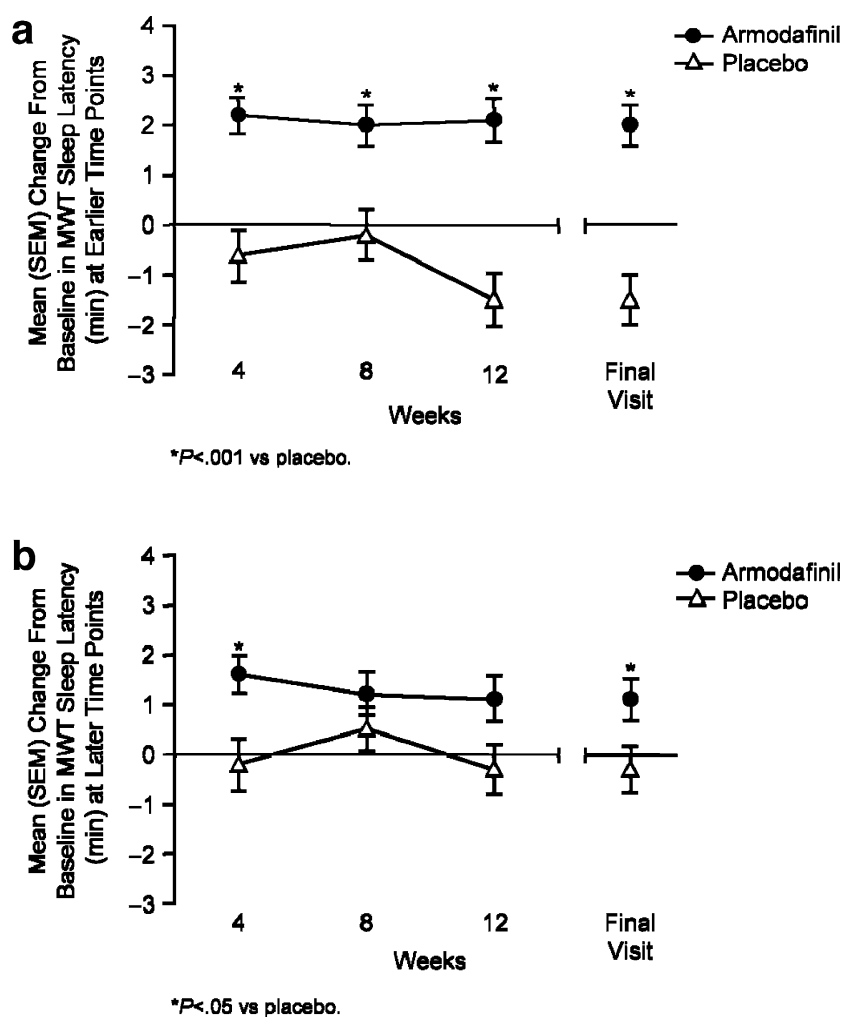

Fig. 2 Mean (SEM) change from baseline in sleep latency from the Maintenance of Wakefulness Test (MWT). a Sleep latencies averaged across the first four tests $(09: 00-15: 00)$. b Sleep latencies averaged across the last three tests (15:00-19:00) 


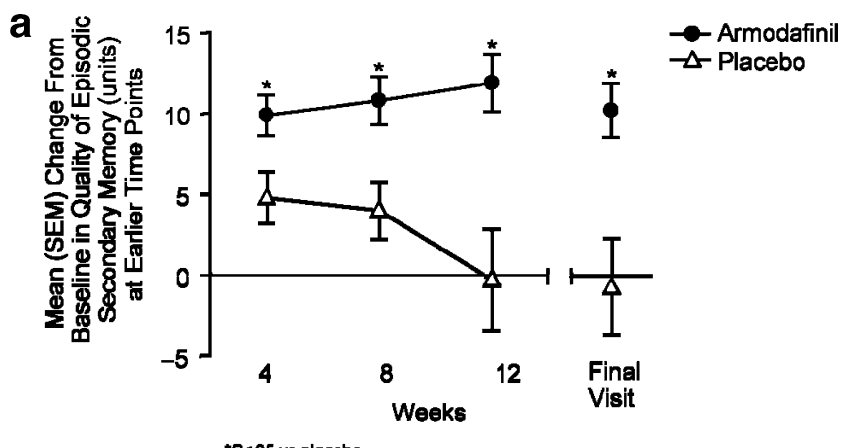

*P<05 va placebo.

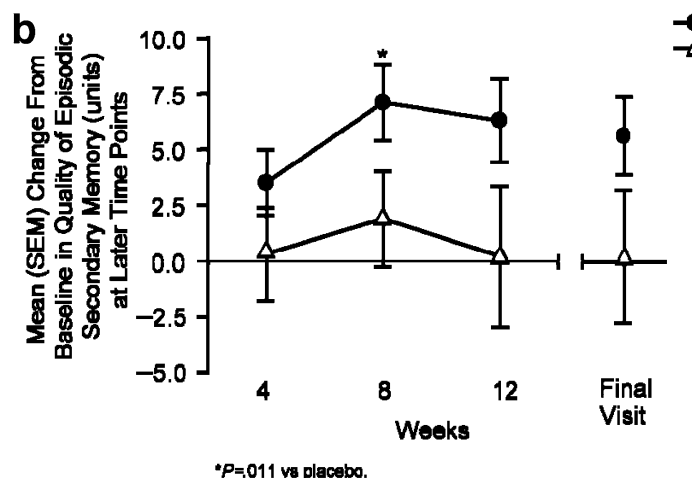

Fig. 3 Mean (SEM) change from baseline in quality of episodic secondary memory. a Scores averaged across the first four tests (09:30-15:30). b Scores averaged across last three tests (15:30-19:30)

\section{Effects on fatigue}

The mean (SD) scores for global fatigue from the BFI at baseline were 4.8 (1.9) and 4.9 (1.9) for the armodafinil and placebo groups, respectively. Armodafinil significantly improved global fatigue at all visits compared with placebo $(P<0.01$; Fig. 5a). Mean scores for worst fatigue in the past $24 \mathrm{~h}$ from the BFI at baseline were nearly identical for the armodafinil and placebo groups (7.2 [2.0] and 7.3 [2.0], respectively) and indicated severe fatigue (score greater than or equal to 7). Armodafinil significantly improved

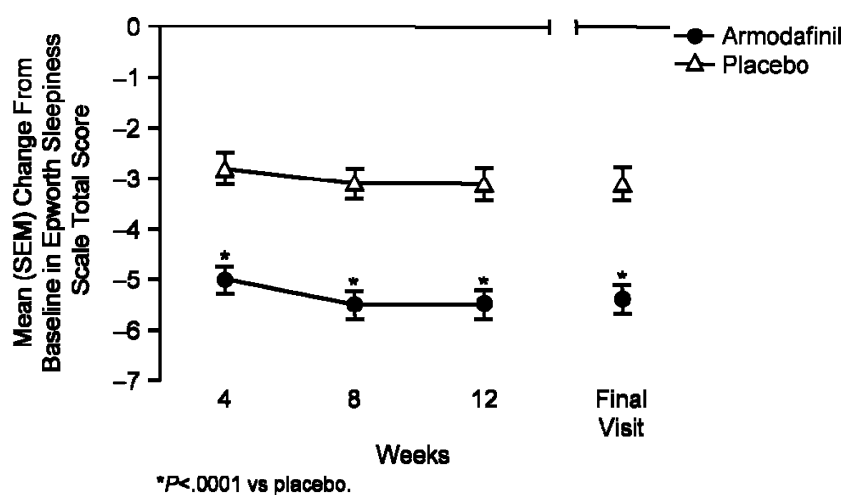

Fig. 4 Mean (SEM) change from baseline in Epworth Sleepiness Scale total score

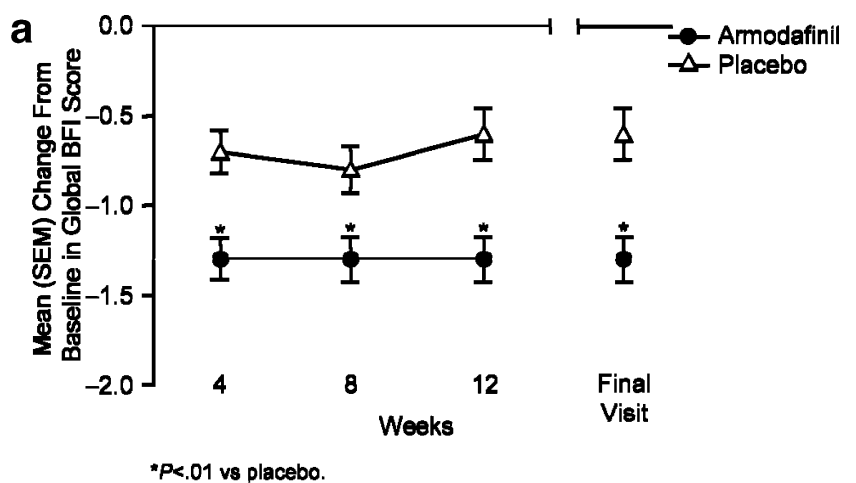

$P<.01$ vs placebo

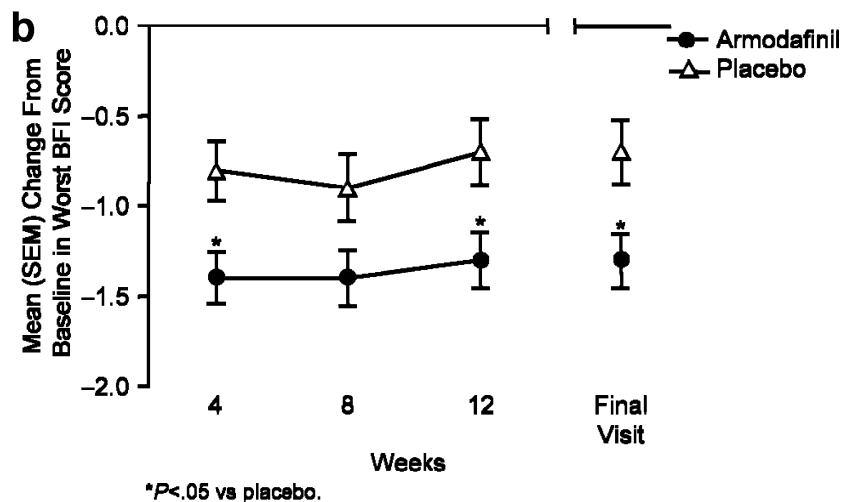

Fig. 5 Mean (SEM) change from baseline in fatigue. a Global fatigue. b Worst fatigue in the past $24 \mathrm{~h}$. BFI Brief Fatigue Inventory

scores for worst fatigue in the past $24 \mathrm{~h}$ at weeks 4 and 12 and at the final visit $(P<0.05$ vs placebo; Fig. $5 b)$, with a trend toward significance at week $8(P=0.056$ vs placebo).

Safety and tolerability

Armodafinil was well tolerated, with a low incidence of adverse events, most of which were mild to moderate in nature. Headache was the most commonly reported adverse event, defined as occurring in greater than or equal to $5 \%$ in either group (Table 2). Serious adverse events were considered by the investigator unlikely to be or not related to armodafinil (ulcerative colitis, $n=1$; migraine, $n=1$; worsening of Axis II disorder and mood disorder, $n=1$; duodenal ulcer hemorrhage, $n=1$ ). One serious adverse event was reported in the placebo group (gastroesophageal reflux disease). The most frequently occurring adverse

Table 2 Adverse events occurring in $\geq 5 \%$ of patients

\begin{tabular}{lll}
\hline Adverse event, $n(\%)$ & Armodafinil $(n=391)$ & Placebo $(n=260)$ \\
\hline Headache & $65(17)$ & $20(8)$ \\
Nausea & $22(6)$ & $10(4)$ \\
Insomnia & $22(6)$ & $3(1)$ \\
Dizziness & $19(5)$ & $4(2)$ \\
Anxiety & $20(5)$ & $2(<1)$ \\
\hline
\end{tabular}


events that led to discontinuation among patients receiving armodafinil were headache $(n=5)$ and nausea $(n=4)$.

There were no clinically significant changes from baseline to final visit in either the armodafinil group or the placebo group for laboratory values, electrocardiogram parameters, or physical examinations. There were also no meaningful changes (clinical or statistical) from baseline to final visit for systolic blood pressure $(0.2$ [14.2] $\mathrm{mmHg}$ for armodafinil vs -1.0 [14.6] $\mathrm{mmHg}$ for placebo), diastolic blood pressure $(0.3$ [9.3] $\mathrm{mmHg}$ for armodafinil vs -1.0 [10.0] $\mathrm{mmHg}$ for placebo), and heart rate (2.3 [9.6] bpm for armodafinil vs 1.4 [9.6] bpm for placebo).

The incidence of patients with newly diagnosed hypertension was less than $1 \%$ in the armodafinil group and less than $1 \%$ in the placebo group. The proportion of patients at risk for hypertension was similar for both treatment groups (18\% of 391 patients, armodafinil group; $16 \%$ of 260 patients, placebo group). The incidence of patients with worsening hypertension was $3 \%$ of 391 patients in the armodafinil group and $2 \%$ of 260 patients in the placebo group.

nCPAP use remained high (approximately $7 \mathrm{~h} /$ night) throughout both of the studies. The duration of nCPAP use at final visit was reduced from baseline by a mean (SD) of -0.3 (0.7) h with armodafinil compared with -0.1 (0.6) h with placebo (a between-group difference of about $12 \mathrm{~min}$; $P<0.0001)$. At final visit, AHI values remained low and were comparable between the armodafinil and placebo groups ( $P>0.05$ vs placebo). In addition, there were no significant changes from baseline in nocturnal PSG sleep variables between the armodafinil and placebo groups $(P>0.05$; Table 3$)$.

\section{Discussion}

Recognizing and effectively managing ES in patients with OSA is essential for clinicians because of the profound impact that ES has on the patients, their families and coworkers, and the general public (e.g., increased risk of traffic accidents) [4-6]. Without appropriate treatment, ES adversely affects cognitive [15, 17], occupational [6], and social functioning [8]. Several studies evaluated in a comprehensive review [11] have shown that there is general improvement in wakefulness with nCPAP therapy, especially in patients with more severe OSA. Nonetheless, residual ES can persist even after effective nCPAP treatment [18], suggesting that there is need for adjunctive therapy to treat ES in these patients.

The present study re-evaluates the findings from two separate studies on the effects of armodafinil in patients with refractory ES associated with OSA and provides additional information regarding secondary analyses, for which the individual studies may not have had adequate statistical power. In the individual studies [26, 27], armodafinil was shown to significantly improve daytime mean sleep latency across the first four tests on the MWT and overall clinical condition as assessed by the CGI-C. In the pooled analysis, armodafinil significantly improved patients' ability to sustain wakefulness as objectively assessed by the MWT. Improvements in mean sleep latency were observed at the first visit at week 4 and were maintained throughout the remainder of the study. The lack of a consistent effect of armodafinil on wakefulness across the last three MWTs $(15: 00,17: 00,19: 00)$ likely reflects the high mean sleep latency (approximately $24.5 \mathrm{~min}$ ) at these times. Behavioral effects, causing poorer performance on the last test of the day, and high intertest variability in sleep latency may have also contributed to this finding [38].

The individual studies also included the clinician's subjective assessment of treatment effect on ES, the CGI-C. Data for the CGI-C, however, were not poolable for this analysis because of substantial differences in the proportion of patients considered responders to placebo between the two studies. In the individual studies, the proportion of patients considered

Table 3 Nocturnal polysomnography parameters ${ }^{\mathrm{a}}$

\begin{tabular}{|c|c|c|c|c|}
\hline \multirow[t]{2}{*}{ Variable (units), mean (SD) } & \multicolumn{2}{|c|}{ Armodafinil $(n=391)$} & \multicolumn{2}{|c|}{ Placebo $(n=260)$} \\
\hline & Baseline & Final Visit & Baseline & Final Visit \\
\hline Latency to persistent sleep (min) & $22.3(26.9)$ & $19.6(20.5)$ & $21.3(24.0)$ & $20.8(21.4)$ \\
\hline Number of arousals, $n$ & $20.0(11.3)$ & $18.5(10.2)$ & $18.7(9.7)$ & $18.4(10.4)$ \\
\hline Number of awakenings, $n$ & $8.8(4.7)$ & $9.2(5.3)$ & $8.7(5.1)$ & $9.6(5.4)$ \\
\hline Sleep efficiency $(\%)$ & $82.4(10.9)$ & $82.0(12.0)$ & $82.0(12.1)$ & $81.4(11.2)$ \\
\hline Wake after sleep onset (min) & $66.6(43.9)$ & $69.1(48.5)$ & $68.7(50.3)$ & $70.2(46.5)$ \\
\hline Stage $1(\%)$ & $11.2(6.4)$ & $10.5(5.5)$ & $10.9(6.1)$ & $10.6(6.2)$ \\
\hline Stage $2(\%)$ & $59.3(9.8)$ & $58.8(11.2)$ & $58.8(10.1)$ & $57.7(11.2)$ \\
\hline Stage 3/4 (\%) & $10.6(9.0)$ & $10.3(9.0)$ & $10.8(9.6)$ & $10.9(10.2)$ \\
\hline REM (\%) & $18.9(6.9)$ & $19.8(7.1)$ & $19.5(7.2)$ & $20.7(8.0)$ \\
\hline
\end{tabular}

REM Rapid eye movement

${ }^{a}$ No significant differences between baseline and final visit were observed. 
responders to armodafinil and placebo was 71 and 53\%, respectively, in one study [26] and 72 and $37 \%$, respectively, in the other study [27].

Cognitive impairment is common in patients with OSA [17]. Although the etiology has not been definitively determined, decreased cortical activity because of impaired arousal or neuronal damage because of chronic intermittent hypoxia are possible causes [16, 39-41]. It is not yet clear what additional interventions may be effective in restoring cognitive function in patients with OSA. Findings from studies evaluating the effects of nCPAP therapy on memory and attention have yielded inconsistent results $[15,16,40$, $42,43]$. Unlike the findings from the individual armodafinil OSA studies [26, 27], the pooled analysis showed that adjunct armodafinil significantly improves long-term memory (i.e., quality of episodic secondary memory) at all study visits compared with placebo, indicating that treatment was associated with a greater ability to recall verbal and visual information. Interestingly, the long-term memory benefit occurred independent of a consistent benefit on attention and concentration. It is important to note that the CDR composite factors in both of the individual armodafinil OSA studies were secondary efficacy variables. Thus, although results from the pooled analysis provide a more precise estimate of the effects on long-term memory, further studies are needed to determine the potential role of armodafinil in improving cognitive function in nCPAPadherent patients with OSA and associated ES.

Armodafinil significantly improved patients' ability to engage in activities of daily living at all visits as measured by the ESS. The patient population studied in this pooled analysis had severe ES (mean ESS score greater than 15) despite effective and regular nCPAP therapy. At the final visit, 49 and $26 \%$ of patients receiving armodafinil and placebo, respectively, had ESS scores less than 10, indicating that nearly half of patients no longer had pathological sleepiness with adjunct armodafinil treatment.

Fatigue may be the presenting complaint of patients with ES [44] and is a common associated symptom [45, 46]. Armodafinil significantly reduced fatigue, a result similar to findings from the individual studies [26, 27]. Armodafinil reduced global fatigue from a baseline value of 4.8 to a final visit value of 3.6. Similarly, worst fatigue in the past $24 \mathrm{~h}$ was reduced from a baseline of 7.2 to 5.8 at final visit. These findings indicate that armodafinil significantly reduced the severity of global fatigue and worst fatigue in the past $24 \mathrm{~h}$ to the mild-to-moderate range of fatigue (BFI score less than 7 [37]).

This pooled analysis shows that armodafinil does not adversely affect nighttime sleep in patients with OSA even with its longer-lasting duration of action compared with modafinil on a milligram-to-milligram basis [24]. Armodafinil was well tolerated; in general, adverse events were rated as mild to moderate in nature, although some patients discon- tinued because of adverse events. There were some minor changes in vital signs; however, these effects were not considered to be clinically significant. The patient population selected for both studies was verified to be adherent to nCPAP therapy; thus, the primary pathology of OSA was being treated in an appropriate and effective manner throughout the course of the two studies. At the final visit, the duration of nCPAP use had decreased minimally from baseline compared with placebo. Average nCPAP use remained high (approximately $7 \mathrm{~h} /$ night) and was effective (i.e., low AHI values). Adjunct armodafinil treatment did not affect arousals.

Findings from the present pooled analysis are limited to patients with OSA who have residual ES despite regular and effective nCPAP therapy and should not be generalized to patients with OSA who are not receiving adequate nCPAP therapy or are not using it regularly. Additionally, the 12-week duration of treatment in these studies limits the applicability of observed results to a longer period of treatment. It should be recognized that armodafinil does not treat the underlying airway obstruction and should not be considered a replacement for nCPAP therapy in patients with OSA. Further research is needed to determine the role armodafinil may have in improving cognitive function and whether the significant reduction in fatigue observed in the pooled analysis will contribute toward improved quality of life in this patient population.

In conclusion, pooled data from two 12-week, doubleblind, placebo-controlled studies showed that once-daily administration of armodafinil significantly improved wakefulness when used as adjunct therapy in nCPAP-adherent patients with residual ES associated with OSA. The effect on wakefulness with armodafinil was maintained throughout the day. Importantly, adjunctive treatment with armodafinil was associated with significant improvements in long-term memory and patients' ability to engage in daily activities. Armodafinil significantly reduced fatigue in the studied population and was well tolerated.

Acknowledgments This study was sponsored by Cephalon, Inc., Frazer, PA. Dr. Roth is a consultant for and has received grant/research support from Cephalon, Inc. Dr. Rippon is an employee of Cephalon, Inc., and Dr. Arora was an employee of Cephalon, Inc. at the time of manuscript preparation. Data were analyzed by the Biometrics Department at Cephalon, Inc. Development of the manuscript was supported by Cephalon, Inc., with significant input and revisions to the satisfaction of all authors. The authors wish to acknowledge Donald G. Buerk, Ph.D., for editorial assistance with the manuscript.

\section{References}

1. American Academy of Sleep Medicine (2005) International classification of sleep disorders: diagnostic and coding manual, 2nd edn. American Academy of Sleep Medicine, Westchester, IL 
2. Olson EJ, Moore WR, Morgenthaler TI, Gay PC, Staats BA (2003) Obstructive sleep apnea-hypopnea syndrome. Mayo Clin Proc 78(12):1545-1552

3. Young T, Palta M, Dempsey J, Skatrud J, Weber S, Badr S (1993) The occurrence of sleep-disordered breathing among middle-aged adults. N Engl J Med 328(17):1230-1235

4. Dincer HE, O'Neill W (2006) Deleterious effects of sleepdisordered breathing on the heart and vascular system. Respiration 73(1):124-130

5. Barbe F, Pericas J, Munoz A, Findley L, Anto JM, Agusti AG (1998) Automobile accidents in patients with sleep apnea syndrome. An epidemiological and mechanistic study. Am J Respir Crit Care Med 158(1):18-22

6. Ulfberg J, Carter N, Edling C (2000) Sleep-disordered breathing and occupational accidents. Scand J Work Environ Health 26 (3):237-242

7. Engleman HM, Douglas NJ (2004) Sleep · 4: sleepiness, cognitive function, and quality of life in obstructive sleep apnoea/hypopnoea syndrome. Thorax 59(7):618-622

8. Kawahara S, Akashiba T, Akahoshi T, Horie T (2005) Nasal CPAP improves the quality of life and lessens the depressive symptoms in patients with obstructive sleep apnea syndrome. Intern Med 44(5):422-427

9. Sharpe M, Wilks D (2002) Fatigue. BMJ 325(7362):480-483

10. Kushida CA, Littner MR, Hirshkowitz M, Morgenthaler TI, Alessi CA, Bailey D, Boehlecke B, Brown TM, Coleman J Jr, Friedman L, Kapen S, Kapur VK, Kramer M, Lee-Chiong T, Owens J, Pancer JP, Swick TJ, Wise MS; American Academy of Sleep Medicine (2006) Practice parameters for the use of continuous and bilevel positive airway pressure devices to treat adult patients with sleep-related breathing disorders. Sleep 29 (3):375-380

11. Giles TL, Lasserson TJ, Smith BJ, White J, Wright J, Cates CJ (2006) Continuous positive airways pressure for obstructive sleep apnoea in adults. Cochrane Database Syst Rev 1:CD001106

12. Bedard MA, Montplaisir J, Malo J, Richer F, Rouleau I (1993) Persistent neuropsychological deficits and vigilance impairment in sleep apnea syndrome after treatment with continuous positive airways pressure (CPAP). J Clin Exp Neuropsychol 15(2):330-341

13. Morisson F, Decary A, Petit D, Lavigne G, Malo J, Montplaisir J (2001) Daytime sleepiness and EEG spectral analysis in apneic patients before and after treatment with continuous positive airway pressure. Chest 119(1):45-52

14. Sforza E, Krieger J (1992) Daytime sleepiness after long-term continuous positive airway pressure (CPAP) treatment in obstructive sleep apnea syndrome. J Neurol Sci 110:21-26

15. Ferini-Strambi L, Baietto C, Di Gioia MR, Castaldi P, Castronovo C, Zucconi M, Cappa SF (2003) Cognitive dysfunction in patients with obstructive sleep apnea (OSA): partial reversibility after continuous positive airway pressure (CPAP). Brain Res Bull 61 (1):87-92

16. Kotterba S, Rasche K, Widdig W, Duscha C, Blombach S, Schultze-Werninghaus G, Malin JP (1998) Neuropsychological investigations and event-related potentials in obstructive sleep apnea syndrome before and during CPAP-therapy. J Neurol Sci 159(1):45-50

17. Naegele B, Thouvard V, Pepin JL, Levy P, Bonnet C, Perret JE, Pellat J, Feuerstein C (1995) Deficits of cognitive executive functions in patients with sleep apnea syndrome. Sleep 18(1):43-52

18. Weaver TE, Maislin G, Dinges DF, Bloxham T, George CFP, Greenberg H, Kader G, Mahowald M, Younger J, Pack AI (2007) Relationship between hours of CPAP use and achieving normal levels of sleepiness and daily functioning. Sleep 30(6):711-719

19. Black JE, Hirshkowitz M (2005) Modafinil for treatment of residual excessive sleepiness in nasal continuous positive airway pressure-treated obstructive sleep apnea/hypopnea syndrome. Sleep 28(4):464-471

20. Pack AI, Black JE, Schwartz JR, Matheson JK (2001) Modafinil as adjunct therapy for daytime sleepiness in obstructive sleep apnea. Am J Respir Crit Care Med 164(9):1675-1681

21. Wong YN, King SP, Simcoe D, Gorman S, Laughton W, McCormick GC, Grebow P (1999) Open-label, single-dose pharmacokinetic study of modafinil tablets: influence of age and gender in normal subjects. J Clin Pharmacol 39(3):281-288

22. Wong YN, Simcoe D, Hartman LN, Gorman S, Laughton W, McCormick GC, Grebow P (1999) A double-blind, placebocontrolled, ascending-dose evaluation of the pharmacokinetics and tolerability of modafinil tablets in healthy male volunteers. J Clin Pharmacol 39(1):30-40

23. Robertson P Jr, Hellriegel ET (2003) Clinical pharmacokinetic profile of modafinil. Clin Pharmacokinet 42(2):123-137

24. Dinges DF, Arora S, Darwish M, Niebler GE (2006) Pharmacodynamic effects on alertness of single doses of armodafinil in healthy subjects during a nocturnal period of acute sleep loss. Curr Med Res Opin 22(1):159-167

25. Harsh J, Hayduk R, Rosenberg R, Wesnes KA, Walsh JK, Arora S, Niebler GE, Roth T (2006) The efficacy and safety of armodafinil as treatment for adults with excessive sleepiness associated with narcolepsy. Curr Med Res Opin 22(4):761-774

26. Hirshkowitz M, Black JE, Wesnes K, Niebler G, Arora S, Roth T (2007) Adjunct armodafinil improves wakefulness and memory in obstructive sleep apnea/hypopnea syndrome. Respir Med 101 (3):616-627

27. Roth T, White D, Schmidt-Nowara W, Wesnes KA, Niebler G, Arora S, Black J (2006) Effects of armodafinil in the treatment of residual excessive sleepiness associated with obstructive sleep apnea/hypopnea syndrome: a 12-week, multicenter, double-blind, randomized, placebo-controlled study in nCPAP-adherent adults. Clin Ther 28(5):689-706

28. US Department of Health and Human Services (1996) Guidance for industry. E6 good clinical practice: consolidated guidance. US Food and Drug Administration, Center for Drug Evaluation and Research, Center for Biologics Evaluation and Research, Rockville, MD

29. Guy W (1976) Clinical Global Impressions. ECDEU assessment manual for psychopharmacology. US Dept of Health and Human Services, National Institute of Mental Health, Rockville, MD, pp 218-222

30. Johns MW (1991) A new method for measuring daytime sleepiness: the Epworth Sleepiness Scale. Sleep 14(6):540-545

31. American Psychiatric Association (2000) Diagnostic and statistical manual of mental disorders, 4th edn. American Psychiatric Association, Washington, DC

32. Poceta JS, Timms RM, Jeong DU, Ho SL, Erman MK, Mitler MM (1992) Maintenance of wakefulness test in obstructive sleep apnea syndrome. Chest 101(4):893-897

33. Doghramji K, Mitler MM, Sangal RB, Shapiro C, Taylor S, Walsleben J, Belisle C, Erman MK, Hayduk R, Hosn R, O'Malley EB, Sangal JM, Schutte SL, Youakim JM (1997) A normative study of the Maintenance of Wakefulness Test (MWT). Electroencephalogr Clin Neurophysiol 103(5):554-562

34. Rechtschaffen A, Kales A (1968) A manual of standardized terminology, techniques and scoring system for sleep stages of human subjects. UCLA Brain Information Service/Brain Research Institute, Los Angeles, CA

35. Keith MS, Stanislav SW, Wesnes KA (1998) Validity of a cognitive computerized assessment system in brain-injured patients. Brain Inj 12(12):1037-1043

36. Wesnes KA, McKeith IG, Ferrara R, Emre M, Del Ser T, Spano PF, Cicin-Sain A, Anand R, Spiegel R (2002) Effects of rivastigmine on cognitive function in dementia with Lewy bodies: a randomised placebo-controlled international study using the 
Cognitive Drug Research computerised assessment system. Dement Geriatr Cogn Disord 13(3):183-192

37. Mendoza TR, Wang XS, Cleeland CS, Morrissey M, Johnson BA, Wendt JK, Huber SL (1999) The rapid assessment of fatigue severity in cancer patients: use of the Brief Fatigue Inventory. Cancer 85(5):1186-1196

38. Arand D, Bonnet M, Hurwitz T, Mitler M, Rosa R, Sangal RB (2005) The clinical use of the MSLT and MWT. Sleep 28 (1):123-144

39. Bartlett DJ, Rae C, Thompson CH, Byth K, Joffe DA, Enright T, Grunstein RR (2004) Hippocampal area metabolites relate to severity and cognitive function in obstructive sleep apnea. Sleep Med 5:593-596

40. Sateia MJ (2003) Neuropsychological impairment and quality of life in obstructive sleep apnea. Clin Chest Med 24(2):249-259

41. Thomas RJ, Rosen BR, Stern CE, Weiss JW, Kwong KK (2005) Functional imaging of working memory in obstructive sleepdisordered breathing. J Appl Physiol 98(6):2226-2234
42. Barnes M, Houston D, Worsnop CJ, Neill AM, Mykytyn IJ, Kay A, Trinder J, Saunders NA, Douglas McEvoy R, Pierce RJ (2002) A randomized controlled trial of continuous positive airway pressure in mild obstructive sleep apnea. Am J Respir Crit Care Med 165(6):773-780

43. Engleman HM, Martin SE, Kingshott RN, Mackay TW, Deary IJ, Douglas NJ (1998) Randomised placebo controlled trial of daytime function after continuous positive airway pressure (CPAP) therapy for the sleep apnoea/hypopnoea syndrome. Thorax 53(5):341-345

44. Shen J, Barbera J, Shapiro CM (2006) Distinguishing sleepiness and fatigue: focus on definition and measurement. Sleep Med Rev 10(1):63-76

45. Lichstein KL, Means MK, Noe SL, Aguillard RN (1997) Fatigue and sleep disorders. Behav Res Ther 35(8):733-740

46. Hossain JL, Reinish LW, Kayumov L, Bhuiya P, Shapiro CM (2003) Underlying sleep pathology may cause chronic high fatigue in shift-workers. J Sleep Res 12(3):223-230 